\title{
Alimentación y calentamiento global: «La larga sombra del ganado» en la prensa española
}

\author{
Nùria Almirón RoIG \\ Universitat Pompeu Fabra \\ nuria.almiron@upf.edu
}

Recibido: 20/06/2012

Aceptado: 18/12/2012

\begin{abstract}
Resumen
La representación mediática del calentamiento global y de sus causas antropogénicas es de enorme relevancia para la aprehensión del tema por parte de la ciudadanía y, por lo tanto, para la correcta percepción de la responsabilidad individual de cada uno de nosotros. Durante años esta representación exhibió un escepticismo irracional que ya se ha abandonado mayoritariamente. Sin embargo, la cobertura periodística de este tema sigue anclada en estructuras mentales que impiden procesar correctamente los hechos reales. Aquí se presenta un caso de estudio que confirma esta tesis en un tema muy vinculado a las decisiones personales: el de la alimentación humana. Las conclusiones reflejan la incapacidad de la prensa de referencia española para comprender y comunicar con responsabilidad el vínculo existente entre comer carne y calentamiento global.
\end{abstract}

Palabras clave: Representación mediática, calentamiento global, alimentación, ganado, prensa española

\section{Food and Global Warming: "Livestock's Long Shadow" in the Spanish Press}

\begin{abstract}
Global warming framing in the news and its anthropogenic causes is of great importance for the apprehension of the subject by the public and, therefore, for the correct understanding of the individual responsibility of each one of us. For years this representation exhibited an irrational scepticism already abandoned by most of the media. However, the coverage of this topic is still rooted in mental structures that prevent processing real facts properly. This paper presents a case of study that confirms this thesis in a subject very related to personal decisions: food. Findings report the inability of the reference Spanish press to understand and communicate responsibly on the link between meat-eating and global warming.
\end{abstract}

Keywords: Framing, global warming, food, livestock, Spanish press

\section{Referencia normalizada}

ALMIRÓN ROIG, Nùria (2013): “Alimentación y calentamiento global: «La larga sombra del ganado» en la prensa española". Estudios sobre el mensaje periodístico. Vol. 19, Núm. 1, págs.: 17-33. Madrid, Servicio de Publicaciones de la Universidad Complutense.

Sumario: 1. Introducción: calentamiento global y cambio climático en los medios de comunicación. 2. Objeto de estudio y metodología. 3. El informe de la FAO "La larga sombra del ganado" sobre el sector pecuario. 4. El interés informativo del informe y las preguntas de esta investigación. 5. Resultados. 7. Conclusiones y discusión. 7. Referencias bibliográficas

\section{Introducción: calentamiento global y cambio climático en los medios de co- municación}

En marzo de 2003 se hizo público en Estados Unidos un informe interno redactado un año antes por el consultor del partido republicano Frank Luntz para la administración de George W. Bush. En una sección del informe titulada Winning the Global Warming Debate, Luntz aportaba argumentos para ayudar a ganar una batalla que los conservadores norteamericanos temían estar perdiendo: la de las causas del calentamiento 
global. En este informe pueden encontrarse las principales ideas repetidas por los escépticos y los negacionistas del cambio climático por causas antropogénicas. En concreto Luntz aconsejaba hacer énfasis en conceptos como la falta de consenso entre los científicos, la incerteza científica, la existencia de expertos rivales, la necesidad de investigar más y todo tipo de argumentaciones dilatorias basadas en promover que sólo la ciencia puede pronunciarse y que los científicos no se ponen de acuerdo ni probablemente se pondrán de acuerdo jamás. El objetivo era perpetuar un estado de duda permanente 1 . Por supuesto, todos estos argumentos obviaban la idea de que existía una masa mayoritaria de científicos a favor de las causas antropogénicas. Pero como es evidente, y el propio informe hacía explícito, no era este un documento que buscara la verdad sobre las causas del calentamiento global sino un memorándum político que definía la estrategia comunicativa y de relaciones públicas que más interesaba al partido republicano. Luntz proponía en las conclusiones del informe cambiar el lenguaje para relativizar el problema (y a sus culpables): "It's time for us to start talking about "climate change" instead of "global warming" [...]. "Climate change" is less frightening than "global warming". (Luntz, 2003: 142). Cabe recordar que los Estados Unidos es uno de los países más contaminantes del planeta.

En realidad el memorándum de Luntz no hacía otra cosa que recomendar una estrategia que había tenido un enorme éxito en la década anterior gracias a la colaboración de la neutralidad periodística. Boykoff y Boykoff (2004) demostraron la sobredimensionada representación que los escépticos y los negacionistas del cambio climático alcanzaron en la prensa occidental, especialmente en la norteamericana, a lo largo de los años noventa. La controvertida norma de la neutralidad periodística había ayudado según este estudio (y también Boykoff y Boykoff, 2007; y Shehata y Hopman, 2012) a amplificar la voz de una minoría que negaba la responsabilidad del ser humano en el cambio climático. A pesar del mayoritario consenso científico a favor de las causas antropogénicas, la prensa occidental estuvo durante años otorgando la misma autoridad y relevancia a ambas posiciones. Como han señalado otros autores, los científicos y los medios de comunicación no eran ni son los únicos actores en este escenario, los intereses económicos y políticos ejercieron y ejercen una considerable presión también. Al respecto afirma Bernando Díaz Nosty: "Frente al avance de la ciencia, se oponen, con frecuencia, intereses ajenos al objeto de la información y de la propia ciencia, descritos por entidades, aparentemente neutrales, financiadas por corporaciones como Exxon-Mobil, que es la que generalmente concita mayor controversia en los análisis de la contaminación mediática desde fuentes interesadas" (Díaz Nosty, 2009: 105).

La cobertura periodística sobre el cambio climático varió posteriormente (Boykoff, 2007), incluso en los medios más hostiles a la teoría de las causas antropogénicas, como era el caso de los medios propiedad del grupo de Rupert Murdoch, News

1 Esta estrategia mimetiza la estrategia de comunicación aplicada por la industria tabaquera durante años para negar el efecto del tabaco en la salud de las personas. Ver en este caso el memorándum interno encargado por The Brown \& Williamson Tobacco Corp en 1969 bajo el título "Smoking and Health Proposal". 
Corp, (McKnight, 2010). Así, desde 2005 se observa una representación mediática progresivamente más madura del tema del calentamiento global en la que las coberturas escépticas o negacionistas de las causas antropogénicas son cada vez más una excepción en los medios de comunicación occidentales, o quedan relegadas a medios de tendencia ideológica muy conservadora (ver por ejemplo Dotson et al, 2012; Mercado, 2012). Incluso algunos han observado el nacimiento de una nueva corriente de comunicación periodística orientada a la sostenibilidad (Díaz Nosty, 2009).

La representación mediática del calentamiento global, o cambio climático, como todo tema científico, es de enorme relevancia para la aprehensión del tema por parte de la ciudadanía. Sin embargo no se ha convertido en objeto de estudio académico hasta muy recientemente en el ámbito anglosajón, especialmente como ejemplo de la importancia del contexto cognitivo subyacente a toda cobertura periodística (ver por ejemplo además de los ya indicados Olausson, 2009; Sonnet, 2010; Olausson, 2011; Nerlich et al, 2012; Mercado, 2012; y muy especialmente Lakoff, 2010). Fuera del ámbito anglosajón la investigación del cambio climático en los medios de comunicación ha sido muy limitada (Díaz Nosty, 2009).

George Lakoff, fundador de la lingüística cognitiva, se refiere particularmente al caso del memorándum de Frank Luntz y al tema del medioambiente para reiterar la importancia de las palabras para crear marcos interpretativos (frames) o estructuras mentales inconscientes a través de las cuales las personas procesamos a su vez la información (Lakoff, 2010). Aunque no existe una teoría unitaria al respecto de este proceso, sí hay un consenso generalizado sobre el hecho que los medios de comunicación enmarcan las informaciones de una determinada manera y estos enfoques ayudan a construir las estructuras mentales a partir de las cuales interpretamos las informaciones -y definimos, en definitiva, los términos del debate público (Tankard, 2001). Según Entman, enmarcar un tema significa seleccionar uno aspectos concretos del mismo y resaltarlos de manera que se promociona una determinada definición del problema, una determinada interpretación de las causas, una determinada evaluación moral y unas determinadas soluciones o salidas posibles (Entman, 1993). Esta definición, interpretación y evaluación del problema no sólo determina los términos del debate público sino también las carencias del mismo.

Lakoff afirma que esta es la razón por la que simplemente aportar hechos reales no lleva a las personas a actuar en la dirección correcta. Las personas deben poseer un sistema mental con estructuras preparadas para entender lo que se les está diciendo. A pesar del cambio radical que la cobertura sobre el calentamiento global ha experimentado en los medios de comunicación desde 2005, Lakoff afirma que los principales marcos mentales o estructuras inconscientes construidos con respecto al tema medioambiental siguen estando hoy impulsados por ideas como las de Luntz, basadas esencialmente en un presupuesto falso: que el medioambiente es algo separado de las personas, que la naturaleza es algo separado de nosotros, cuando en realidad nosotros somos una parte inseparable de ella. Lakoff argumenta que la visión neoconservadora ha sido y sigue siendo mucho más eficaz que la visión progresista a la hora de crear las estructuras mentales que actualmente procesan el debate medioambiental en las mentes de la mayoría de las personas (Lakoff, 2010). 
En este artículo se presenta un caso de estudio que apoya la tesis de Lakkof al mostrar cómo estas estructuras mentales siguen ancladas en marcos que impiden procesar correctamente los hechos, en un tema con profundas implicaciones para el cambio climático y muy vinculado a las decisiones personales: el de la alimentación humana.

\section{Objeto de estudio y metodología}

El objeto de estudio aquí es la cobertura realizada por la prensa de referencia española de un muy singular informe sobre el sector pecuario publicado por la Organización de Naciones Unidas para la Alimentación y la Agricultura (FAO) en 2006: "La larga sombra del ganado. Problemas ambientales y opciones" ("Livestock's long shadow. Environmental Issues and Options", en su versión en inglés). En concreto se analiza la cobertura y el discurso realizado por la prensa española con respecto al citado informe de la FAO desde la publicación de este, a finales de noviembre de 2006, hasta marzo de 2012.

Por prensa de referencia se considera aquí a los periódicos de información general con referente impreso no gratuito más leídos según la Oficina de Justificación de la Difusión. Los periódicos elegidos fueron El Pais, El Mundo, ABC, La Vanguardia y El Periódico de Cataluña. El corpus empírico de la investigación lo constituyen los artículos de cualquier género periodístico que se han podido localizar a través de la base de datos Factiva y las hemerotecas digitales de los periódicos y que se centran o mencionan el citado informe de la FAO.

La metodología de análisis empleado es doble. Por una parte utiliza el análisis de contenido clásico para identificar y clasificar las piezas localizadas y analizar si estas abordan o no determinadas cuestiones contenidas en el informe de la FAO. Por otra parte se aplica el análisis crítico del discurso desarrollado por autores como Norman Fairclough (1995), Teun Van Dijk (1996), Sigried, Jäger (2001), Ruth Wodak y Michael Meyer (2003), y Xavier Giró (2007). Con este enfoque se han obtenido las principales proposiciones explícitas e implícitas de cada pieza analizada para responder a las cuestiones cualitativas que se formulan y obtener así una macroproposición o síntesis ideológica de cada pieza.

El objeto es descubrir cual es el enfoque o representación mediática del informe y sus contenidos en la prensa estudiada atendiendo a las estrategias interpretativas utilizadas por los periodistas. Para formularnos las preguntas/objetivos de la investigación se han tomado como punto de partida las estrategias interpretativas (framing strategies) propuestas por Todd Gitlin: el uso de fuentes oficiales, la trivialización de las acciones de los actores con visiones opuestas, la atención en el suceso y no en el tema en su vertiente más amplia y el énfasis en una sola de las versiones argumentativas (en Mercado, 2012).

\section{El informe de la FAO "La larga sombra del ganado" sobre el sector pecuario} La Organización de Naciones Unidas para la Alimentación y la Agricultura (FAO) es el brazo de Naciones Unidas para temas de agricultura y alimentación y está especialmente concentrada en intentar combatir el hambre en el mundo. Publica anualmente cientos de documentos sobre temas relacionados con esta cuestión (como 
cosechas, enfermedades, alimentación, bosques, nutrición, sostenibilidad, infraestructuras y calidad de vida rural, etc.) en forma de libros, artículos, informes o memorándums. En 2011 su base de datos en Internet ofrecía 5.730 documentos accesibles sólo para ese año y el año de publicación del informe que aquí nos ocupa, 2006, arroja 2.607 documentos publicados por esta organización. Algunos de sus informes reciben mucha atención por parte de los medios de comunicación, que utilizan este organismo de Naciones Unidas como fuente de autoridad para temas de alimentación y agricultura en el mundo, sobre todo en lo concerniente a los países en vías de desarrollo.

El informe que aquí se aborda, "La larga sombra del ganado", constituye un estudio con unas conclusiones sin precedentes en cuanto a claridad y contundencia expositiva. Sus autores analizan el impacto del sector ganadero sobre el medio ambiente y lo sitúan entre los más contaminantes del planeta. En particular, afirman que al ganado se deben el 9\% del total de las emisiones de dióxido de carbono, el 37\% de las de metano y el $65 \%$ de las emisiones de óxido nitroso. Con respecto específicamente al cambio climático, aseguran que la participación en el calentamiento global del ganado asciende a cerca del 18 por ciento. Este porcentaje supone que su participación sería superior a la contribución del sector transporte en todo el mundo. Posteriormente se generó cierta controversia al saberse que en la comparación ganado versus transporte no se había partido de cifras equiparables, pues si bien se tuvieron en cuenta para las emisiones del ganado a todos los gases producidos desde el campo hasta la mesa, en lo relativo al sector del transporte no existe un indicador global similar, y sólo se dispone de los datos del combustible quemado en cada traslado (y no, por ejemplo, de las emisiones derivadas de la extracción petrolífera, la producción de coches o la construcción de carreteras). Este error sería utilizado en contra del estudio, pero no invalida para nada las conclusiones del mismo: que el sector pecuario es tremendamente contaminante y uno de los más contaminantes para el planeta.

"La larga sombra del ganado" supone la primera vez que se evalúa de forma específica la contribución del sector pecuario al calentamiento global. Hasta entonces se disponía de muchos indicadores indirectos pero no de datos directos. Por ejemplo, el Panel Intergubernamental de Naciones Unidas para el Cambio Climático (IPCC) ya citaba como principales causas de emisión de gases de efecto invernadero los siguientes datos de 2004: a la energía (25,9\%), la industria (1,4\%), la deforestación $(1,4 \%)$, la agricultura $(13,5 \%)$, el transporte $(13,1 \%)$, el sector residencial y los edificios comerciales $(7,9 \%)$ y los residuos y aguas residuales (2,8\%) (IPCC, 2007: 36). Dentro de la agricultura se incluía el impacto del sector pecuario pero es preciso tener

2 El informe fue realizado por los miembros del equipo LEAD (Livestock, Environment and Development) de la FAO. El proyecto LEAD está apoyado por el Banco Mundial, la Unión Europea, el Ministerio de Asuntos Exteriores francés, el Ministerio para la Cooperación y el Desarrollo Económico alemán, el Departamento para el Desarrollo Internacional del Reino Unido, la Agencia estadounidense para el Desarrollo Internacional, la Agencia para el Desarrollo Internacional danesa, la Agencia para la Cooperación y el Desarrollo suiza, el Fondo Internacional para el Desarrollo Agrícola (IFAD) y la Organización para la Alimentación y la Agricultura (FAO) de Naciones Unidas. 
en cuenta que buena parte de las emisiones del resto de sectores son debidas también a la explotación ganadera. El informe de la FAO proporcionaba cifras mucho más concretas sobre este impacto, como por ejemplo las siguientes (FAO, 2006):

- El sector pecuario representa el principal uso antropogénico de la tierra (la superficie total dedicada pastos o cultivos para ganado es del $70 \%$ de toda la tierra agrícola del planeta).

- El sector pecuario es uno de los principales responsables del agotamiento del agua. La FAO estima que el sector pecuario participa en al menos una cuarta parte del consumo total de agua (sólo en riego de cultivos para piensos ya se dedica el $7 \%$ del total del consumo mundial de agua, y esto no incluye el agua para abrevar el ganado, para el regadío de pastos, para la elaboración de carne y leche, etc.).

- La ganadería extensiva, practicada en zonas como la Amazonía, es la razón fundamental de la deforestación en el planeta.

- La ganadería intensiva o sistemas industriales a gran escala, la dominante en el planeta (en algunos casos ya la única forma de explotación de determinados animales, como las aves de corral) y la que mayores desechos contaminantes genera, supone el $80 \%$ del crecimiento del sector pecuario.

- En la mayor economía del mundo, Estados Unidos, la ganadería es la responsable del 55\% de la erosión, el 37\% de la aplicación de plaguicidas y el 50\% del volumen de antibióticos consumido en todo el país.

- El sector pecuario es uno de los causantes principales de la pérdida de biodiversidad en el planeta (el ganado representa cerca del $20 \%$ del total de los animales terrestres y ocupa una vasta superficie de lo que alguna vez fueron hábitats silvestres).

El informe situaba además el impacto de la ganadería en el planeta en un contexto económico y nutricional. Así, afirmaba (FAO, 2006):

- En términos numéricos simples el ganado resta más valor del suministro total de alimentos del que proporciona (consume más proteína comestible para los humanos de las que produce).

- A pesar de ser un gran contaminante el sector pecuario tiene un peso relativamente pequeño en la economía mundial. Sólo genera el 1,4\% del PIB mundial (2005). Si bien en el caso de algunos países pobres la producción pecuaria puede representar hasta el 25\% del PIB, como es el caso de Mongolia, esto no necesariamente significa que la ganadería sea una vía para la reducción de la pobreza.

- En términos nutricionales se observa un enorme contraste: mientras las proteínas animales pueden ayudar a mejorar enormemente las dietas de los países más pobres, en los países ricos un elevado número de enfermedades no transmisibles se asocia a la elevada ingestión de alimentos de origen animal.

Los datos ofrecidos para este último punto relativo a la nutrición son especialmente chocantes. Si bien hay 864 millones de personas en el mundo que, según el informe, podrían solucionar sus problemas de desnutrición o malnutrición gracias a los produc- 
tos de origen animal, el número de personas afectadas por sobrepeso u obesidad (en los que el abusivo consumo de productos de origen animal es una de las principales causas) eran estimados en 2006 en 1.300 millones por la FAO. De hecho, el informe llega literalmente a afirmar que "los daños ambientales originados por la actividad ganadera podrían disminuir considerablemente si la gente rica disminuyera el consumo excesivo de productos de origen animal" (FAO, 2006: 304). Mientras en un país como la India se consume 5 kilogramos de carne por persona al año en los Estados Unidos se llega a los 123 kilogramos por persona al año. En España, uno de los países con mayor consumo de carne del mundo, se superan los 118 kilogramos por persona al año. ${ }^{3}$

En realidad, todos los datos aportados por el informe ponen en evidencia que la ganadería tiene un coste medioambiental insostenible para el planeta, que no garantiza la seguridad alimentaria (el ganado consume más proteínas de las que produce, como se indicaba) y que existe un enorme desequilibrio entre el consumo de proteína animal en el mundo. Además, el sector pecuario aumenta la demanda general de alimento y los precios de los cultivos y los insumos agrícolas -lo que perjudica especialmente a los países pobres-. Y esto no hará más que aumentar debido al incremento de demanda de carne que se espera provenga de países emergentes como China y al crecimiento económico y demográfico en general futuros. De modo que la previsión es que el impacto del sector ganadero en el medioambiente aumente.

A pesar de lo anterior, el informe se torna muy cauto cuando llega a las recomendaciones finales:

- Asume que la expansión de la demanda de productos ganaderos aumentará en el futuro y alerta de que el sector debe crecer reduciendo los impactos ambientales para ser sostenible.

- La solución a la contradicción anterior (cómo es posible reducir los impactos a mayor demanda si no se consiguen reducir con la demanda actual) se deja en manos de la tecnología y de los consumidores conscientes y sin problemas económicos (capaces de presionar a los políticos para que se proteja el medioambiente y de asumir un incremento de los precios).

- Es preciso eliminar las distorsiones de los precios de tierras, aguas y piensos utilizados por la ganadería industrial para que estos reflejen la verdadera escasez de los recursos. Ello hará que los usemos con mayor eficiencia y ahorro.

- Es necesario aceptar que la intensificación, y quizás también la industrialización de la producción pecuaria, constituye una característica inevitable de los resultados a largo plazo del proceso de cambio estructural que experimenta el sector.

En favor de la brevedad, no se abordan aquí todos los detalles ni matices que se incorporan en las recomendaciones -especialmente con respecto al rol del sector pecua-

3 Es significativo tener en cuenta que si bien la tendencia general en los países desarrollados en los últimos años ha mostrado una ligera reducción en el consumo de carne por persona al año, la FAO indica que las causas de esta moderación no tienen que ver con una mayor concienciación medioambiental entre la población sino con la crisis económica. La moderación del consumo de carne sería por tanto una tendencia coyuntural. 
rio en las economías más pobres- pero se hace preciso resaltar la enorme contradicción de base de sus términos. Por un lado se describe un sector tremendamente destructivo para el medio ambiente y consumido abusivamente por los ciudadanos ricos -cuyo número no hará más que aumentar en un futuro en términos absolutos-. Por otro lado, sin embargo, las recomendaciones omiten apuntar a las causas del problema directamente señaladas por los datos: que una parte minoritaria de la población ingiere demasiados productos de origen animal y que la tendencia de las poblaciones emergentes es a imitarla. Sorprendentemente el crecimiento demográfico y el consumo abusivo no se abordan en las recomendaciones finales del informe. Se promueve en cambio la aceptación de la producción intensiva esperando que la tecnología resuelva el enorme problema de los desechos y obviando por completo la cuestión ética de fondo: el sufrimiento y dolor que la explotación industrial intensiva irremediablemente produce en los animales de granja, seres sintientes que son tratados como objetos.

El informe termina con un llamamiento a actuar con urgencia recordando que "la información, la comunicación y la educación tienen una responsabilidad clave para promover una mayor voluntad de acción" (FAO, 2006: 318) y que debe cambiarse la percepción inadecuada que tiene la sociedad civil del problema para hacerla más consciente de los riesgos ambientales del mismo.

Extraer el principal mensaje del informe es sencillo pues la FAO ha preparado un resumen del mismo para los medios de comunicación en el que destaca como conclusión general del estudio el reto de reconciliar dos demandas que entran en conflicto entre ellas: la demanda de productos animales y las necesidades medioambientales.

\section{El interés informativo del informe y las preguntas de esta investigación}

El interés informativo de "La larga sombra del ganado" es evidente no sólo por los datos precisos con los que se cuantifica un problema de enorme magnitud hasta entonces sólo evaluado indirectamente por organismos oficiales y no oficiales ${ }^{4}$, sino también porque, a pesar del pragmatismo políticamente correcto de las conclusiones, el informe es claro al respecto de la causa final del problema y de su solución. Se considera aquí que este informe posee todas las cualidades para merecer una destacada atención mediática, de entre todas las publicaciones de la FAO, por el hecho de proporcionar a los ciudadanos una herramienta individual para solucionar lo que posiblemente es el problema más grave que afecta a nuestras sociedades: el deterioro del planeta del que depende nuestra supervivencia.

El análisis del informe nos permite formular las cuestiones que debemos responder para desvelar el enfoque interpretativo de la cobertura periodística del mismo. El informe de la FAO reitera el importante rol de los medios de comunicación (y la educación) a la hora de trasladar a la sociedad civil una percepción adecuada del alcance

${ }^{4}$ Después de este informe diversas organizaciones no gubernamentales han alertado también específicamente del impacto que el consumo de carne tiene sobre el medioambiente (ver por ejemplo Worldwatch Institute, 2009 o The Environmental Working Group-EWG, 2011). 
del problema. Para comprobar si este rol se ejerce se respondió a estas preguntas para cada artículo analizado:

1. ¿Se explica u ofrece una sintesis del estudio de la FAO? El informe y el tema es de gran relevancia para los ciudadanos. ¿La cobertura dada al informe ofrece suficiente información al lector?

2. ¿Se trasladan objetivamente las conclusiones y recomendaciones del estudio? El informe aborda en las conclusiones y recomendaciones finales los principales problemas que hay que combatir. ¿Se plasman claramente en la información periodística? Con esta y la anterior pregunta se pretende observar si la cobertura realizada es completa o si sólo se informa parcialmente del asunto.

3. ¿Se aborda la causa final del problema? El informe establece un vínculo directo entre comer productos de origen animal y el deterioro medio ambiental del planeta. Se afirma además que gran parte del problema podría resolverse si los ciudadanos ricos redujeran drásticamente su ingesta de carne. El informe evita plantear el problema sólo en estos términos pero es muy claro al respecto de este vínculo y causa final del problema. ¿Lo son los artículos analizados?

4. ¿Se presentan todos los argumentos o posiciones ante el tema? Se observa aquí qué fuentes se citan al cubrir la información sobre el informe. ¿Se aplica la neutralidad periodística presentando todas las posiciones por igual o se pone énfasis sólo en unas determinadas voces?

5. ¿Se aborda el informe y el tema con seriedad? ¿Se trivializa la cuestión o se trata con el rigor necesario que requiere el tema?

6. ¿Se aborda el informe y el tema con espiritu crítico? El informe adolece de una serie de contradicciones que el quehacer periodístico debería detectar. A pesar de señalar claramente la causa de problema (el consumo, afectado por las variables de desigualdad social y demografía), no la tiene en consideración en las recomendaciones. A pesar de plantear un escenario muy negativo con tendencias a empeorar, las recomendaciones no recogen la "urgencia" (en términos de la propia FAO) del problema, otorgando un peso muy importante de la solución a la tecnología del futuro. Se obvia además la presión de la industria pecuaria sobre el consumidor y los intereses económicos en general que subyacen detrás del tema. ¿Los artículos analizados señalan algunas de estas contradicciones y ausencias?

7. ¿Se aborda el componente ético del problema? El informe no aborda el problema ético que subyace tras la cuestión: el enorme dolor psíquico y físico que infringimos a miles de millones de seres vivos en las explotaciones ganaderas industriales de todo el planeta. ¿Lo hace la cobertura periodística?

8. ¿El texto/autor del texto toma partido en general? ¿El periodista se posiciona explícita o implícitamente a favor o en contra del informe? ¿Promueve la concienciación o relativiza el problema? Es esta una valoración del artículo en general, obtenida a partir de la macroproposición general identificada para cada pieza.

9. ¿Se emplaza al consumidor a actuar y a ejercer su poder como consumidor? El informe de la FAO afirma que una de las claves, si no la principal, para la solución del problema se encuentra en las decisiones personales que tomamos los ciudadanos ricos con respecto al modo de alimentarnos. El informe reitera el importante rol de los me- 
dios de comunicación (y la educación) a la hora de hacer que la sociedad civil se movilice y ejerza el enorme poder que tiene como consumidora. ¿Ejercen este rol, directa o indirectamente, las piezas analizadas?

10. ¿El periodista demuestra estar informado sobre la cuestión? Para valorar el grado de conocimiento del asunto por parte del periodista se evalúa si hay evidencias en el texto de que su autor se haya leído el informe de la FAO y si demuestra conocer el contexto y el alcance real del problema (por ejemplo, citando otras fuentes que aportan datos similares a la FAO u enlazando con otros artículos relacionados, etc.).

\section{Resultados}

Para el período de tiempo estudiado, un total de cinco años, se localizaron a través de la base de datos Factiva un total de 1.388 artículos en los que se mencionaba a la FAO en los cinco periódicos españoles estudiados. La revisión de todos estos artículos se completó con las hemerotecas digitales de todas las cabeceras con excepción de $E l P e$ riódico de Catalunya, cuya hemeroteca digital no permite búsquedas avanzadas y para el que sólo se utilizó Factiva. A través de este proceso se identificaron 16 piezas en las que se mencionaba directa o indirectamente (sin mencionar el título) el informe "La larga sombra del ganado" publicado a finales de noviembre de 2006 por la FAO.

Tabla 1. Artículos que cubren o mencionan el informe "La larga sombra del ganado" de la FAO entre 2007 y 2011

\begin{tabular}{|c|c|c|c|c|c|c|c|}
\hline Cabecera & Fecha & Género & Sección & Título & Autor & Lugar & $\begin{array}{l}\text { Pala- } \\
\text { bras }\end{array}$ \\
\hline El País & $1-4-2007$ & Reportaje & Sociedad & $\begin{array}{l}\text { "Las vacas se parecen a } \\
\text { los coches más de lo que } \\
\text { se cree" }\end{array}$ & Andrea Rizzi & Madrid & 939 \\
\hline El País & 24-7-2009 & Reportaje & Sociedad & $\begin{array}{l}\text { "Lo más ecológico... } \\
\text { ¿que no haya vacas? }\end{array}$ & Antía Castedo & $\mathrm{Nd}$ & 2.043 \\
\hline El País & $28-3-2010$ & Noticia & Sociedad & $\begin{array}{l}\text { "La ONU admite otro } \\
\text { fallo en un estudio sobre } \\
\text { cambio climático" }\end{array}$ & Antía Castedo & $\begin{array}{l}\text { B a r c e - } \\
\text { lona }\end{array}$ & 391 \\
\hline$A B C$ & $16-6-2009$ & Noticia & Gente & $\begin{array}{l}\text { "Paul McCartney y sus } \\
\text { 'lunes sin carne"" }\end{array}$ & Reuters i AFP & Londres & 436 \\
\hline$A B C$ & 25-12-2009 & Noticia & $\begin{array}{l}\text { Ciencia y } \\
\text { Tecnología }\end{array}$ & $\begin{array}{l}\text { "Argentina monta "vacas } \\
\text { mochileras" contra el } \\
\text { cambio climático" }\end{array}$ & Efe & $\begin{array}{l}\text { Buenos } \\
\text { Aires }\end{array}$ & 397 \\
\hline$A B C$ & $23-3-2010$ & Noticia & $\begin{array}{l}\text { Canal Na- } \\
\text { tural }\end{array}$ & "Vacas más verdes" & L.A. Gámez & $\mathrm{Nd}$ & 543 \\
\hline$A B C$ & $30-4-2010$ & Noticia & Agencias & $\begin{array}{l}\text { "Carne producida en la- } \\
\text { boratorios abre la puerta } \\
\text { a un futuro sin matade- } \\
\text { ros" }\end{array}$ & Efe & $\mathrm{Nd}$ & 610 \\
\hline El Mundo & $20-4-2007$ & Opinión & Ecología & "Pedos de vaca" & Alfredo Merino & $\mathrm{Nd}$ & 467 \\
\hline El Mundo & $11-9-2007$ & Noticia & Solidaridad & 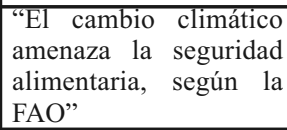 & Europa Press & Madrid & 521 \\
\hline
\end{tabular}




\begin{tabular}{|c|c|c|c|c|c|c|c|}
\hline El Mundo & $8-9-2008$ & Noticia & Ciencia & $\begin{array}{l}\text { "La ONU recomienda } \\
\text { reducir el consumo de } \\
\text { carne para luchar contra } \\
\text { el cambio climático" }\end{array}$ & Agencias & Londres & 452 \\
\hline El Mundo & 11-11-2009 & Noticia & Europa & $\begin{array}{l}\text { "McCartney defenderá } \\
\text { en la Eurocámara que } \\
\text { hay que comer menos } \\
\text { carne" }\end{array}$ & Efe & Bruselas & 192 \\
\hline El Mundo & $21-2-2010$ & Noticia & $\begin{array}{l}\text { Castilla y } \\
\text { León }\end{array}$ & $\begin{array}{l}\text { "Extractos de ajo i acei- } \\
\text { tes esenciales para redu- } \\
\text { cir los gases de vacas y } \\
\text { ovejas" }\end{array}$ & J.A.O. & León & 362 \\
\hline El Mundo & $17-10-2011$ & Noticia & Economía & $\begin{array}{l}\text { "Los gobiernos echan } \\
\text { mano de los impuestos } \\
\text { más extravagantes" }\end{array}$ & Rocio Bergillos & Madrid & 510 \\
\hline El Mundo & 28-11-2011 & Noticia & Agricultura & $\begin{array}{l}\text { "De los gases de las } \\
\text { vacas al uso de fertili- } \\
\text { zantes" }\end{array}$ & $\begin{array}{l}\text { Miguel G. Co- } \\
\text { rral }\end{array}$ & $\mathrm{Nd}$ & 291 \\
\hline $\begin{array}{l}\text { La Van- } \\
\text { guardia }\end{array}$ & $9-2-2009$ & Noticia & Tendencias & $\begin{array}{l}\text { "Contra la contamina- } \\
\text { ción, menos carne" }\end{array}$ & Rafael Poch & Berlín & 546 \\
\hline $\begin{array}{l}\text { La Van- } \\
\text { guardia }\end{array}$ & $31-5-2011$ & Noticia & Tendencias & $\begin{array}{l}\text { "La mujer contamina } \\
\text { menos; El Hombre gasta } \\
\text { más energía: viaja en } \\
\text { coche, sale más e ingiere } \\
\text { más carne" }\end{array}$ & $\begin{array}{l}\text { Antonio Cerri- } \\
\text { llo }\end{array}$ & $\mathrm{Nd}$ & 933 \\
\hline
\end{tabular}

Una síntesis del análisis cuantitativo ofrece los siguientes resultados:

- Sólo se localizó un artículo que cubriera la publicación del informe poco después de la aparición de éste. Otros dos artículos se dedicaban al error comparativo que incluía el informe. El grueso de artículos abordan el tema del informe y utilizan el informe, en solitario o junto a otras fuentes, para documentar el tema. La mayoría de estos artículos se publican años después de la aparición del informe.

- El periódico que más artículos ha publicado mencionando el informe es $E l$ Mundo (7), mientras que no hemos podido localizar ningún artículo mencionando el informe en El Periódico de Catalunya.

- El periódico que aborda en mayor profundidad el informe, y que más palabras en total le dedica, es El País, con el 35\% del total de palabras analizadas. Los dos únicos reportajes identificados sobre el tema son de este periódico.

- La sección en la que se ubican más artículos que mencionan el informe es la de Sociedad (37\% de los artículos), seguida de las de Ciencia y Ecología (26\% conjuntamente).

Una síntesis del análisis cualitativo ofrece los siguientes resultados:

\section{„Pregunta 1 y 2: El informe es trasladado muy parcialmente a la opinión pú- blica}

Sólo cinco artículos ofrecen una descripción correcta del informe y no hay ningún artículo que traslade las conclusiones del informe y sus recomendaciones. Incluso en los únicos tres artículos que se centran en el informe, la información es muy incompleta. 
En el caso del reportaje de El País que cubre la publicación del informe (el único artículo identificado que lo hace), las conclusiones y recomendaciones del mismo no son trasladadas correctamente. Los otros dos artículos que se centran en el informe lo hacen para restarle autoridad, sin describir el informe y sus conclusiones lo suficiente para que el lector tenga toda la información. En un caso, $A B C$, incluso se publica un artículo en contra de las conclusiones del informe sin antes haber cubierto su publicación y, por lo tanto, sin haber trasladado previamente una descripción de sus contenidos al lector. El resto de artículos que mencionan el informe lo hacen colateralmente y sin ofrecer una descripción completa del informe.

\section{„Pregunta 3: La mayoría de los artículos presenta al ganado como el culpa- ble último del problema}

Más de la mitad de los artículos no abordan la causa final del problema (comer carne en exceso por parte de una porción minoritaria de la población del planeta) y los que la abordan (siete artículos) en ocasiones es para relativizarla. Sólo en cinco textos se presenta claramente el consumo como la causa final, el resto de artículos presenta al ganado como el culpable.

En el caso de El País, el periódico que da mayor cobertura al informe, el reportaje que cubre su publicación llega a afirmar " ¿Cómo puede el ganado hacer tanto daño con su digestión?" achacando la culpabilidad a la vacas, el estiércol y las flatulencias, y no a su masificación por causa de la enorme demanda de carne. No obstante, el periódico rectifica esta visión criminalizadora del ganado en un artículo posterior donde directamente afirma que "reducir el consumo de carne en los países ricos sería un método rápido y eficaz para que cada ciudadano contribuya a frenar el calentamiento global".

En el caso de $A B C$, los artículos que abordan la causa final lo hacen para relativizarla o incluso negarla. La solución para este periódico no tiene que ver con el consumo sino con la tecnología.

En el caso de El Mundo sólo en dos de sus siete artículos dedicados al tema aborda la causa final abiertamente mientras en el caso de La Vanguardia los dos únicos artículos encontrados sobre el tema abordan la causa última del problema con claridad.

\section{»Pregunta 4: En la inmensa mayoría de los artículos no se aplica la neutrali- dad periodística al tema}

La neutralidad periodística que da igual valor a todas las posiciones no se encuentra presente en la muestra de artículos estudiada. Esto sucede bien porque en una minoría de casos (dos) se da voz a todas las posiciones, pero no se tratan estas por igual, o bien porque no sé da voz a todas las posiciones. Pero la ausencia de neutralidad no siempre viene regida por una justificación argumental (dar más peso a la posición mejor fundamentada). El periódico $A B C$ es quien menos neutralidad justificada demuestra. En uno de los artículos ataca una campaña vegetariana promovida por el exBeattle Paul McCartney sin ofrecer otros argumentos que la opinión del autor del texto, oculto tras una "Agencias". En otro artículo $A B C$ ataca las conclusiones del comité de expertos redactores del informe de la FAO dando más credibilidad a la opinión contraria proferida en una conferencia por un solo científico. En los dos artículos restantes analizados de este periódico, la opción tecnológica es vista como la solución final, sin mención a otras opciones, sin aportar argumentos creíbles sobre la via- 
bilidad real de la misma -aunque reconociendo que se trata de opciones caras y no operativas todavía-. Entre los otros periódicos se han encontrado no obstante piezas donde no existe neutralidad de partida pero tampoco manipulación objetiva.

\section{lidad \\ »Pregunta 5: La mayoría de los artículos aborda el problema real con frivo-}

En general se detecta una tendencia general a dar un trato frívolo a la contaminación producida por los animales o a las opciones vegetarianas que podrían paliarla. Encontramos mofas de los animales: "Nada mal las vaquitas", "Los de los coches no son los únicos tubos de escape que amenazan el planeta”, “¡Ojo a las vacas!” (El País), "vacas mochileras" $(A B C)$, "pedos de vaca" (El Mundo); o mofas y desprestigio de las personas que apoyan una opción vegetariana para combatir el cambio climático: "la última ocurrencia del ex Beatle" o "el día elegido para animar al populacho a convertirse en vegetarianos ocasionales" $(A B C)$. En cambio cuando se abordan las soluciones presentadas como científicas o el error de comparación del informe de la FAO el tono es de extrema seriedad. La Vanguardia es el único periódico en que todas las piezas analizadas, dos, abordan la contaminación producida por el ganado evitando frivolizar con el asunto.

\section{»Pregunta 6 y 7: El espíritu crítico y el componente ético están absolutamente ausentes de la cobertura del informe}

Ninguno de los artículos muestra espíritu crítico con respecto a los puntos fuertes y débiles del informe de la FAO. O bien se describe el informe sin mencionar sus contradicciones, o bien se critica al informe sin fundamento. El problema ético de fondo, la explotación intensiva de seres vivos a los que se obliga a vivir de forma miserable, no es abordado por ninguno de los artículos directamente y sólo indirectamente por un articulo, que menciona el tema al referirse a las declaraciones del presidente, y premio Nobel de la Paz, del panel sobre el cambio climático de Naciones Unidas.

„Pregunta 8: Una gran mayoría de los textos toma partido, pero para relativizar el problema

De los 16 artículos identificados, cinco no toman partido ni explícita ni implícitamente (tres artículos de El Mundo y los dos de La Vanguardia) y nueve, la mayoría, lo toman relativizando el problema. De entre estos últimos, cinco relativizan el problema explícitamente a través de restar valor al informe por su error de comparación (El País), ridiculizar a quien se preocupa $(A B C)$, directamente negar los resultados del informe o asegurar que la tecnología lo resolverá ( $A B C$ y El Mundo). Sólo dos artículos (publicados en El País) toman partido para concienciar del problema, aunque lo hacen implícitamente.

»Pregunta 9: La inmensa mayoría de los textos no moviliza a los lectores a actuar o incluso los desmoviliza

Sólo tres artículos invitan al lector a actuar pero siempre indirectamente, a través de declaraciones de terceros. En cambio la mayoría puede incluso desmovilizar al lector en la media en que se presenta al problema como solucionable con la tecnología, se ridiculiza al activismo provegetariano o incluso directamente se niega el que el lector pueda tener nada que ver con el tema del calentamiento global a través de su consumo de carne. 


\section{»Pregunta 10: La inmensa mayoría de los artículos demuestran falta de in- formación sobre el tema}

Sólo un artículo demuestra estar bien documentado, pero no es significativo pues cubre un aspecto muy parcial y reducido del tema (el del error de comparación). El resto de textos no muestran conocer bien el informe ni parecen conocer la existencia de otras fuentes que corroboran el informe. En general el nivel de información de los autores de los textos es modesto o muy insuficiente, llegándose a difundir errores propios de la cultura popular. Por ejemplo, diversos artículos relacionan la expulsión de metano de las vacas con las flatulencias, cuando en realidad estos animales expelen principalmente el metano a través del hocico en forma de eructos -como se desprende del propio informe de la FAO, por otro lado-. Hasta 5 artículos cometen este error. En cambio, ningún texto menciona las causas del incremento de metano expulsado por estos rumiantes, que no se debe sólo al gran número de ganado criado por los humanos sino también a la sobrealimentación a la que se les somete, para aumentar y mantener el mayor peso posible (a más alimentación, más fermentación entérica en sus estómagos y mayor producción de metano) y al tipo de alimentación que se les obliga a ingerir.

\section{Conclusiones y discusión}

La conclusión de esta investigación es lógicamente que la prensa española de referencia no prestó atención al informe de la FAO a pesar de la contundencia y claridad de sus resultados al respecto de la relación entre consumo de carne y calentamiento global; y a pesar de la apelación de la misma FAO a la responsabilidad de los medios de comunicación en dar a conocer y explicar estos resultados. En general es posible afirmar que la prensa estudiada no parece tener interés por el tema en general, que no es aquí el del calentamiento global (por el que sí tiene mucho interés, por supuesto) sino el del rol que un aspecto específico de nuestros hábitos alimentarios tiene en el calentamiento global.

Sin embargo esta falta o poco interés no es equiparable en todos los periódicos analizados. Mientras en La Vanguardia y El País existe cierto grado de responsabilidad en su cobertura (a pesar de relegar el tema a secciones como Sociedad o Tendencias), en El Mundo y $A B C$ es donde mayor resistencia parece haber a aceptar los resultados del informe. La fe en la tecnología y la irracional criminalización del ganado promueve entre estos dos últimos periódicos el mayor número de coberturas frívolas y el menor grado de responsabilidad periodística, o mejor dicho, el mayor grado de irresponsabilidad social, habida cuenta de la relevancia del tema que nos ocupa para el futuro de nuestro planeta y de nuestra propia especie.

La inevitable pregunta es ¿por qué sucede esto? ¿Acaso no es un dato de máximo interés conocer que algo tan sencillo como reducir el consumo de carne puede tener un impacto directo en el medio ambiente? ¿Qué impide a estos periódicos abordar el tema con mayor atención, documentación, claridad y responsabilidad?

Para responder a estas cuestiones se propone aquí volver a Lakoff. En términos generales, los medios de comunicación estudiados serían incapaces de situar los datos en su contexto por lo que este autor denomina "hipocognición", o ausencia en la mente 
de las ideas que permiten construir estructuras mentales capaces de entender las últimas consecuencias de lo que se está diciendo. La ausencia de estas ideas mentales inconscientes impedirían aquí construir las estructuras mentales que llevan a hacer comprender con claridad el vínculo existente entre comer carne y calentamiento global. $\mathrm{O}$, lo que es lo mismo, el vínculo entre un hábito individual y cotidiano muy arraigado y el grave deterioro del planeta a nivel medioambiental y social. ¿A qué podría deberse la ausencia de estas ideas mentales o hipocognición entre los periodistas? Apuntamos aquí a tres causas principales: una económica, otra psicológica y una tercera filosófica o moral.

La presión económica de la industria agroalimentaria y el peso de ésta en la sociedad (con enormes inversiones en marketing y publicidad para vender sus productos o financiar investigaciones) sin duda tiene un considerable impacto en nuestras construcciones mentales, y por lo tanto en nuestras decisiones. Pero probablemente mayor es el peso psicológico que arrastra el ancestral acto de comer carne, profundamente vinculado a la masculinidad y la virilidad en las sociedades occidentales, como apuntan algunos estudios (ver Potts y Parry, 2010).

Finalmente el abusivo cultivo de la mente y el intelecto en la hipertecnificada sociedad moderna, en detrimento del espíritu y el crecimiento interior, impide desarrollar una ética profunda y genuina en la que pueda florecer la compasión por los otros seres vivos que comparten con nosotros este planeta, humanos y no humanos. Y añadir, así, a los factores medioambientales (deterioro del planeta), sociales (enfermedades de la alimentación, inseguridad alimentaria y desequilibrio social) y económicos (costes de todo lo anterior) el componente moral que nos haga reaccionar.

\section{Referencias bibliográficas}

BOYKOFF, Maxwell T. (2007):'Flogging a dead norm? Newspaper coverage of anthropogenic climate change in the United States and United Kingdom from 2003 to 2006". Area, Vol. 39 No. 4, pp. 470-481, 2007.

BOYKOFF, Maxwell T., BOYKOFF, Jules M. (2007): "Climate change and journalistic norms: A case-study of US Mass-media coverage". Geoforum, 38 (6) 11901204 ,

BOYKOFF, Maxwell T., BOYKOFF, Jules M. (2004): "Balance as bias: Global warming and the US prestige press". Global Environmental Change, 14 (2) 125-136.

DÍAZ NOSTY, Bernardo (2009): "Cambio climático, consenso científico y construcción mediática. Los paradigmas de la comunicación para la sostenibilidad". Revista Latina de Comunicación Social, 64. 99-119.

DOTSON, Devin M.; JACOBSON, Susan K.; KAID, Lynda Lee y CARLTON, J. Stuart (2012): "Media Coverage of Climate Change in Chile/ A Content Analysis of Conservative and Liberal Newspapers". Environmental Communication/ A Journal of Nature and Culture, Volume 6, Issue 1, 64-81.

ENTMAN, Robert (1993): "Framing: toward clarification of a fractured paradigm". Journal of Communication 43(4), pp. 51-58. 
FAIRCLOUGH, Norman (1995): Critical Discourse Analysis. Boston, Addison Wesley.

FAO (2006): “Livestock's Long Shadow. Environmental Issues and Options. Roma: FAO [Versión en castellano: "La larga sombra del ganado. Problemas ambientales y opciones". Roma: FAO, 2009].

GIRÓ, Xavier (2007): "Enfoques analíticos críticos sobre el discurso de la cobertura informativa de conflictos". En TELLESCHI, Tiziano; SANDOVAL FORERO, Eduardo Andrés (coords.): Espacio y tiempo en la globalización. Una visión de la transparencia en la información. Universita' di Pisa y Comisión Estatal para el Acceso a la Información Pública del Estado de Sinaloa, Toluca (México), pp. 199-220.

IPCC (2007): "Climate change 2007. Synthesis Report". Naciones Unidas, Panel Intergubernamental sobre el Cambio Climático.

JA"GER, Sigfried (2001): "Discourse and knowledge: theoretical and methodological aspecs of a critical discourse and dispositive analysis". En RUTH WODAK \& MICHAEL MEYER (ed.): Methods of Critical discourse Analysis, London: Sage.

LAKOFF, George (2010): "Why it Matters How We Frame the Environment". Environmental Communication: a Journal of Nature and Culture, vol. 4, $\mathrm{n}^{\circ} .1, \mathrm{pp} .70$ 81.

LUNZ, Frank (2003): "The Environment: A Cleaner, Safer, Healthier America". Memorándum de Frank Luntz, The Luntz Research Companies. Copia obtenida por el Environmental Working Group y disponible en: http://www.sindark.com/NonBlog/Articles/LuntzResearch_environment.pdf [consultado el 27 de marzo de 2012].

MCKNIGHT, David (2010): "A change in the climate? The journalism of opinion at News Corporation”, Journalism 11(6) 693-706.

MERCADO, María Teresa (2012): "Media Representations Of Climate Change In The Argentinean Press", Journalism Studies, 13:2, 193-209.

NERLICH, Brigitte; FORSYTH, Richard; CLARKE, David (2012): "Climate in the News/ How Differences in Media Discourse Between the US and UK Reflect National Priorities", Environmental Communication/ A Journal of Nature and Culture, Vol. 6, n. 1, 44-63.

OLAUSSON, Ulrika (2009): "Global warming-global responsibility? Media frames of collective action and scientific certainty". Public Understand. Science 18, 421436

OLAUSSON, Ulrika (2011): "We're the Ones to Blame"/ Citizens' Representations of Climate Change and the Role of the Media", Environmental Communication/A Journal of Nature and Culture, Vol. 5, n.3, 281-299.

POTTS, Annie y PARRY, Jovian (2010): "Vegan Sexuality: Challenging Heteronormative Masculinity through Meat-free Sex". Feminism \& Psychology, Vol. 20, n. $1,53-72$. 
SHEHATA, Adam y HOPMANN, David Nicolas (2012): "Framing Climate Change", Journalism Studies, 13:2, 175-192.

SONNET, John (2010): "Climates of risk: A field analysis of global climate change in US media discourse, 1997-2004". Public Understand. Science 19(6), 698-716.

TANKARD, James W. JNR (2001): “The Empirical Approach to the Study of Media Framing'. En: REESE, Stephen D.; GAND,Y Oscar H. y GRANT ,August E. (Eds): Framing Public Life: perspectives on media and our understanding of the social world, Mahwah, NJ: Lawrence Erlbaum Associates, pp. 95-106.

THE BROWN \& WILLIAMSON TOBACCO CORP (1969): "Smoking and Health Proposal". Bates No. 690010927/0935, Legacy Tobacco Documents Library, University of California, San Francisco: http://legacy.library.ucsf.edu/action/document/page? tid $=$ ogy93f00\&page $=4$ [Consultado el 27 de marzo de 2012].

THE ENVIRONMENTAL WORKING Group-EWG (2011): “A Meat Eater's Guide to Climate Change". En The Environmental Working Group: http://www.ewg.org/ [Fecha de consulta: 1 de abril de 2012].

VAN DIJK, Teun. 1996. “Opiniones e ideologías en la prensa”. Voces y Culturas, n. 10-II semestre pp. 9-50.

WODAK, Ruth and MEYER, Michael (2003): Métodos de Análisis Crítico del Discurso. Barcelona: Gedisa.

WORLDWATCH INSTITUTE (2009): "Livestock and climate change". Worldwatch Institute. Disponible en www.worldwatch.org/ww/livestock. [consultado el 19 de junio de 2012). 